\title{
Indications for Dzyaloshinskii-Moriya interaction at the Pd/Fe interface studied by in situ polarized neutron reflectometry
}

\author{
Sina Mayr,,${ }^{1,2, *}$ Jingfan Ye,,${ }^{1, \dagger}$ Jochen Stahn, ${ }^{2}$ Birgit Knoblich, ${ }^{3}$ Oliver Klein, ${ }^{3}$ Dustin A. Gilbert, ${ }^{4}$ \\ Manfred Albrecht, ${ }^{3}$ Amitesh Paul, ${ }^{1}$ Peter Böni, ${ }^{1}$ and Wolfgang Kreuzpaintner $\odot^{1,5, \#}$ \\ ${ }^{1}$ Physik-Department E21, Technische Universität München, James-Franck-Straße 1, 85748 Garching, Germany \\ ${ }^{2}$ Paul Scherrer Institut, CH-5232 Villigen PSI, Switzerland \\ ${ }^{3}$ Experimentalphysik IV, Institut für Physik, Universität Augsburg, Universitätsstraße 1 Nord, 86159 Augsburg, Germany \\ ${ }^{4}$ Department of Materials Science and Engineering, University of Tennessee, Knoxville, Tennessee 37996, USA \\ ${ }^{5}$ Guangdong Technion-Israel Institute of Technology, 241 Daxue Road, Jinping District, Shantou, 515063 Guangdong Province, China
}

(Received 16 April 2019; revised manuscript received 29 September 2019; published 8 January 2020)

\begin{abstract}
Using in situ polarized neutron reflectometry, the depth-resolved evolution of the magnetism and structure in a $\mathrm{Pd} / \mathrm{Fe} / \mathrm{Pd}$ trilayer thin film is measured during growth. The initial film structure of $\mathrm{Pd} / \mathrm{Fe}$ shows a small proximity-induced magnetism in the underlayer and a magnetization in the Fe layer of $\approx 1.6 \mu_{\mathrm{B}}$ per Fe atom, less than the expected bulk value of $2.2 \mu_{\mathrm{B}}$. Deposition of the Pd capping layer initially follows an islandlike growth mode with subsequent coalescence. With increasing Pd deposition the Fe moment and the proximity-induced magnetism in the Pd capping layer decrease. After final deposition of the Pd capping layer, the magnetic profile is structurally and magnetically symmetric across the Fe layer, with magnetism induced in Pd up to $0.92 \mathrm{~nm}$ from the interface. Throughout the $\mathrm{Pd}$ deposition the $\mathrm{Pd} / \mathrm{Fe} / \mathrm{Pd}$ trilayer structure is becoming increasingly symmetric, a fact which points to a Dzyaloshinskii-Moriya interaction as a likely cause of the observed magnetic behavior.
\end{abstract}

DOI: 10.1103/PhysRevB.101.024404

\section{INTRODUCTION}

Thin films and heterostructures exhibit a variety of fascinating electronic, magnetic, and optical properties [1-9] and are therefore indispensable for scientific and technological applications. Most of the functional characteristics of layered structures are determined by the processes taking place during their preparation by thin-film deposition, viz., when the sample structure, material stoichiometry, and defect population are defined. For a fundamental understanding of magnetism on an atomic scale, it is therefore crucial to investigate the evolution of magnetism in thin layers and heterostructures in situ during growth and to correlate the magnetic properties with the corresponding microstructure. In situ studies are particularly critical when the system possesses proximity effects such as induced magnetism.

The Fe/Pd thin-film system is known to show strong proximity-induced magnetism and has been widely studied experimentally [10-17] and theoretically [15-20]. In the $\mathrm{Fe} / \mathrm{Pd}$ system, proximity-induced magnetism has been reported in the Pd layer up to $2 \mathrm{~nm}$ [10] from the Fe interface, with an induced magnetization of $0.3 \mu_{\mathrm{B}}-0.4 \mu_{\mathrm{B}} /$ atom $^{\mathrm{Pd}}$ at the interface $[13,15,20]$.

Further, at the interface between a heavy-metal (HM) element with strong spin-orbit coupling and a ferromagnetic transition metal (FM), magnetic spin structures develop chiral

\footnotetext{
*sina.mayr@psi.ch

†jingfan.ye@frm2.tum.de

${ }^{\ddagger}$ wolfgang.k@gtiit.edu.cn
}

domain walls, spirals, or skyrmions due to the interfacial Dzyaloshinskii-Moriya interaction (IDMI) [21-28]. Previous works have suggested that the IDMI is not related to the proximity-induced magnetization [29]. Accordingly, in systems that show both IDMI and proximity-induced magnetism, it is not clear what the magnetic structure at the interface will be, especially when the surfaces are asymmetric. Furthermore, the HM layer can be used to generate strong spin-orbit torques, arising from the spin-Hall effect [30], allowing the manipulation of these interfacial magnetic structures without affecting the induced magnetization away from the interface. This makes such systems highly promising candidates to realize high-speed and energy-efficient memory devices and offers tremendous opportunities for research and technological applications.

In this work, the evolution of magnetism in a polycrystalline $\mathrm{Pd}(11 \mathrm{~nm}) / \mathrm{Fe}(0.41 \mathrm{~nm}) / \operatorname{Pd}(72 \mathrm{~nm}) / \mathrm{Si}$ (substrate) trilayer heterostructure is investigated. Films were grown by conventional direct current (dc) magnetron sputtering and investigated in situ by polarized neutron reflectometry (PNR). The stepwise deposition of $\mathrm{Pd}$ onto a $\mathrm{Pd} / \mathrm{Fe}$ bilayer system initially occurs by island growth before becoming a continuous film. The induced magnetisms in both the $\mathrm{Pd}$ capping layer and Pd underlayer are separately resolved and quantified as Pd is deposited. Surprisingly, the magnetism in the Pd films is initially asymmetric, with the thin capping layer having an induced moment of $0.6 \mu_{\mathrm{B}}$ per atom, while the induced magnetism in the underlayer is $0.2 \mu_{\mathrm{B}}$ per atom ( $\sim 65 \%$ smaller). Increasing the Pd capping layer thickness motivates symmetry in the $\mathrm{Fe}$ interfaces and results in a magnetic symmetry. The emergence of magnetic symmetry 
TABLE I. PNR fit parameters for the Si substrate, the Pd seed, and the Fe layer. For the number density $n$, also the ratio $n / n_{\text {bulk }}$ is given.

\begin{tabular}{|c|c|c|c|c|}
\hline & Thickness $d$ & Number density $n$ & $n / n_{\text {bulk }}$ & rms roughness $\sigma$ \\
\hline $\mathrm{SiO}_{2}$ layer & $d^{\mathrm{SiO}_{2}}=0.99\left({ }_{-0.56}^{+0.51}\right) \mathrm{nm}$ & $n^{\mathrm{SiO}_{2}}=2.58\left({ }_{-0.26}^{+0.41}\right) \times 10^{22} \mathrm{~cm}^{-3}$ & 0.96 & $\sigma^{\mathrm{SiO}_{2}}=0.58\left({ }_{-0.19}^{+0.24}\right) \mathrm{nm}$ \\
\hline Pd seed & $d^{\mathrm{Pd}_{\text {seed }}}=71.93\left({ }_{-0.79}^{+1.09}\right) \mathrm{nm}$ & $n^{\mathrm{Pd}_{\text {seed }}}=5.66\left({ }_{-0.05}^{+0.16}\right) \times 10^{22} \mathrm{~cm}^{-3}$ & 0.83 & $\sigma^{\mathrm{Pd}_{\text {sed }}}=1.76\left({ }_{-0.10}^{+0.06}\right) \mathrm{nm}$ \\
\hline Fe layer & $d^{\mathrm{Fe}}=0.41\left({ }_{-0.12}^{+0.11}\right) \mathrm{nm}$ & $n^{\mathrm{Fe}}=7.97\left(\begin{array}{c}+0.31 \\
-0.29\end{array}\right) \times 10^{22} \mathrm{~cm}^{-3}$ & 0.94 & $\sigma^{\mathrm{Fe}}=1.73( \pm 0.06) \mathrm{nm}$ \\
\hline
\end{tabular}

based on the structural symmetry of the $\mathrm{Pd} / \mathrm{Fe} / \mathrm{Pd}$ interfaces implies that Dzyaloshinskii-Moriya interactions are the likely origin of the observed behavior.

\section{EXPERIMENTAL DETAILS}

Films of $\operatorname{Pd}(11 \mathrm{~nm}) / \mathrm{Fe}(0.41 \mathrm{~nm}) / \operatorname{Pd}(72 \mathrm{~nm})$ were grown by dc magnetron sputtering on a $2 \times 2 \mathrm{~cm}^{2} \mathrm{Si}(001)$ substrate in an ultrahigh-vacuum chamber $\left(p_{\text {base }}=5 \times 10^{-9}\right.$ mbar $)$ installed at the Apparatus for Multi Option Reflectometry (AMOR) neutron reflectometer at Swiss Spallation Neutron Source (SINQ), Paul Scherrer Institute, Villigen [31]. The deposition system is equipped with three sputtering sources with $2^{\prime \prime}(5.1 \mathrm{~cm})$ diameter which can be positioned above the sample surface without breaking the vacuum. This allows the Pd and Fe to be deposited without exposing the sample to atmosphere or realigning it in the neutron beam. Control over the layer thicknesses is achieved via the opening times of a deposition shutter.

Sample growth was performed at room temperature without any heating or cooling of the substrate. Pd was sputtered from a $99.99 \%$ pure target using an ultrahigh purity $(7 \mathrm{~N})$ Ar working gas at a pressure of $3.37 \times 10^{-3}$ mbar and with $20 \mathrm{~W}$ of dc sputtering power, resulting in a deposition rate of $0.33 \mathrm{~nm} / \mathrm{s}$. Fe was deposited at an Ar pressure of $4.69 \times$ $10^{-3}$ mbar from a $99.95 \%$ pure sputtering target at $20 \mathrm{~W}$, resulting in a deposition rate of $0.13 \mathrm{~nm} / \mathrm{s}$. The $\mathrm{Pd}$ underlayer and $\mathrm{Fe}$ layer were grown in single steps, while the 11-nm Pd capping layer was grown in 18 steps with approximately $0.6 \mu \mathrm{g} / \mathrm{cm}^{2}$ of Pd deposited per step.

PNR is a grazing-incidence neutron scattering technique with high spatial magnetic and nuclear sensitivity [32,33]. The evolution of the nuclear density and magnetization was followed in situ, with PNR measurements being performed after each of the deposition steps.

Specular neutron reflectometry captures the depth-resolved average nuclear and magnetic scattering length density (SLD) and, accordingly, is sensitive to in-plane variations in the sample. Features such as island growth manifest as a reduction in the expected SLD. Using the nuclear SLD for each atom, the atomic number density can be determined. The magnetic SLD can be converted to magnetization $(\mathrm{A} / \mathrm{m})$ by dividing it by $2.853 \times 10^{-6} \mathrm{~mA}^{-1} \AA^{-2}$, which can then be transformed to magnetic moment per atom using the number density.

Except for a very few demonstration cases, PNR measurements have all been performed on films after growth (i.e., ex situ), and thus, emergent behavior during thin-film growth could not be investigated by PNR. Facilitated by recent developments [34,35], PNR can now also be applied as an in situ technique ( $i$ PNR). By growing samples at the neutron beamline, $i$ PNR allows the evolution of the structural and magnetic properties of the entire film to be captured as a function of the layer thicknesses, one deposition step after the other. For clarity, $i$ PNR captures the magnetic and nuclear depth profile of the entire film at each measurement, with spatial sensitivity along the thickness of the sample. Therefore, the evolution of the depth profile as a function of deposited Pd thickness is resolved. The major advantage of $i$ PNR for the study presented here lies in the simultaneous accessibility to the magnetic properties in $\mathrm{Pd}$ on both sides of the Fe layer.

To perform these measurements in a reasonable time frame, prototype focusing Selene neutron optics [31,36,37] were used, with a neutron wavelength band of 4-10 $\AA$ and a neutron beam divergence of $1.6^{\circ}$. With these settings the resolution increases from $\frac{\Delta q}{q} \approx 4.5 \%$ in the regime of total reflection to a quasistatic value of $\frac{\Delta q}{q} \approx 2.3 \%$ for $q_{z} \gtrsim$ $0.2 \mathrm{~nm}^{-1}$. Beam polarization was realized by the transmittance of the neutrons through an $m=4.2 \mathrm{Fe} / \mathrm{Si}$ multilayer polarizer with a logarithmic spiral shape [38]. The neutron polarization was selected by an rf spin flipper. In-vacuum guide fields perpendicular to the scattering plane maintained the neutron polarization up to the sample position. A magnetic field of $70 \mathrm{mT}$ was applied in plane to saturate the sample using permanent magnets. The $i$ PNR data acquisition times were approximately 35 min for each spin direction, which at the given vacuum quality was sufficient to rule out any contamination from the residual gas.

A theoretical model was fitted to the experimental $i$ PNR data using the SimulReflec software [39]. The errors of the fit parameters are estimated by a $5 \%$ increase over the optimum figure of merit approximately equal to $\sum\left|\ln R_{\mathrm{fit}}-\ln R_{\text {meas }}\right|$ on independent variation of a single parameter [40], where $R_{\mathrm{fit}}$ is the fitted reflectivity and $R_{\text {meas }}$ is the measured reflectivity.

The theoretical models include a natural $\mathrm{SiO}_{2}$ layer on the surface of the $\mathrm{Si}$ substrate on which the $\operatorname{Pd}(11 \mathrm{~nm}) / \mathrm{Fe}(0.41 \mathrm{~nm}) / \operatorname{Pd}(72 \mathrm{~nm})$ trilayer structure is grown. These fit parameters are given in Table I. The $\mathrm{Fe}$ layer was simulated such that its structural and magnetic thicknesses were identical. For Pd, the best agreement with the experimental data was obtained by allowing a region of up to $0.92 \mathrm{~nm}$ from the Fe interface to carry a magnetic moment. This magnetic regime agrees well with previous reports on induced magnetism in the first two [15] to four monolayers [13]. For the fitting process the 0.92-nm regions on either side of the Fe layer were split into four equally thick regimes of $0.23 \mathrm{~nm}$, each carrying its own magnetization. These magnetic regimes are identified as $\mathrm{Pd}-4, \mathrm{Pd}-3, \mathrm{Pd}-2$, and $\mathrm{Pd}-1$ (below the $\mathrm{Fe}$ layer) and $\mathrm{Pd}+1, \mathrm{Pd}+2, \mathrm{Pd}+3$, and 


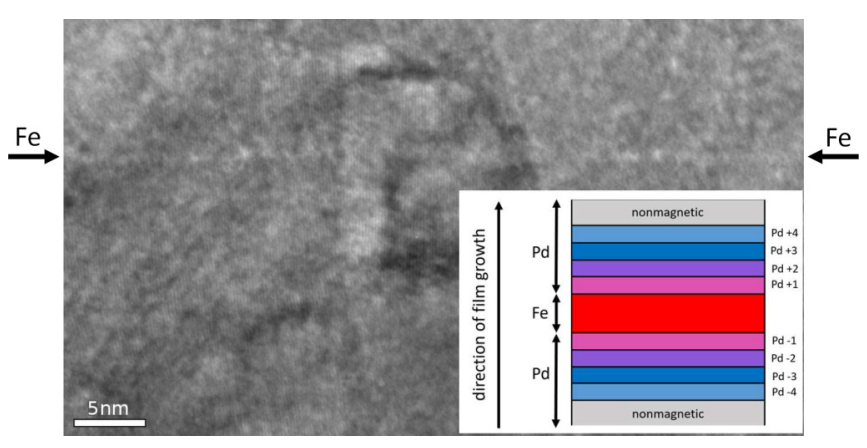

FIG. 1. TEM image showing the Fe layer buried in Pd. The sharp interfaces between $\mathrm{Fe}$ and $\mathrm{Pd}$ are visible. The inset shows the model for the magnetic structure of the sample used for the fitting process with the Fe layer (red) and the adjacent Pd sublayers, Pd-1 to Pd-4 and $\mathrm{Pd}+1$ to $\mathrm{Pd}+4$.

$\mathrm{Pd}+4$ (covering the $\mathrm{Fe}$ ) with $\mathrm{Pd}-1$ and $\mathrm{Pd}+1$ being at the interface with the $\mathrm{Fe}$ and $\mathrm{Pd}-4$ and $\mathrm{Pd}+4$ being the interface with the assumed nonmagnetic part of the Pd layers. The theoretical model is shown in the inset of Fig. 1. It is noted that attempts to simulate the PNR data on the basis of a different model resulted in a less perfect agreement with the $i$ PNR data (viz., models based on no magnetization in the Pd [15] or larger regimes of induced magnetization) or in nonphysical values of the Fermi vectors for models based on the assumption of Ruderman-Kittel-Kasuya-Yosida-like (RKKY) oscillatory magnetic behavior in the Pd.

Transmission electron microscopy (TEM) measurements were performed ex situ on the fully grown sample using a field-emission JEOL $2100 \mathrm{~F}$ microscope at $200 \mathrm{kV}$, equipped with an ultrahigh resolution pole piece. The point-to-point resolution specified by the manufacturer is $0.194 \mathrm{~nm}$. Note that a value for the interfacial root-mean-square (rms) roughness is obtained from the decay of the $i$ PNR reflectivity curves. It statistically describes the deviation from the mean interface level over the illuminated sample area of $20 \times 2 \mathrm{~mm}^{2}$ but does not allow the lateral in-plane length scales at which an interface "appears" rough to be assessed. For this, off-specular $i$ PNR data would be required, which is not obtainable if the Selene neutron optics are applied. Consequently, the TEM results are used to locally identify interface and layer quality and the microstructure of the fully grown sample.

\section{RESULTS AND DISCUSSION}

Images obtained by transmission electron microscopy (Fig. 1) show that the Pd and Fe layers are polycrystalline. The mass contrast of the $\mathrm{Fe}$ and $\mathrm{Pd}$ allows the identification of the $\mathrm{Fe}(0.41 \mathrm{~nm})$ layer as a horizontal white line in Fig. 1. The sharp boundary of the Fe layer confirms the high-quality growth of the film with minimal interdiffusion.

The $i$ PNR data overlaid with best-fit theoretical reflectivity curves as a function of deposition step $i$ are shown in Fig. 2. The magnetic depth profiles which fit best the experimental reflectivity of each measured deposition step are shown in Fig. 3. Here, the magnetic moments are plotted in units of $\mu_{\mathrm{B}}$. The evolution of the nuclear (e.g., structural) and magnetic depth profiles versus deposition step is discussed below.

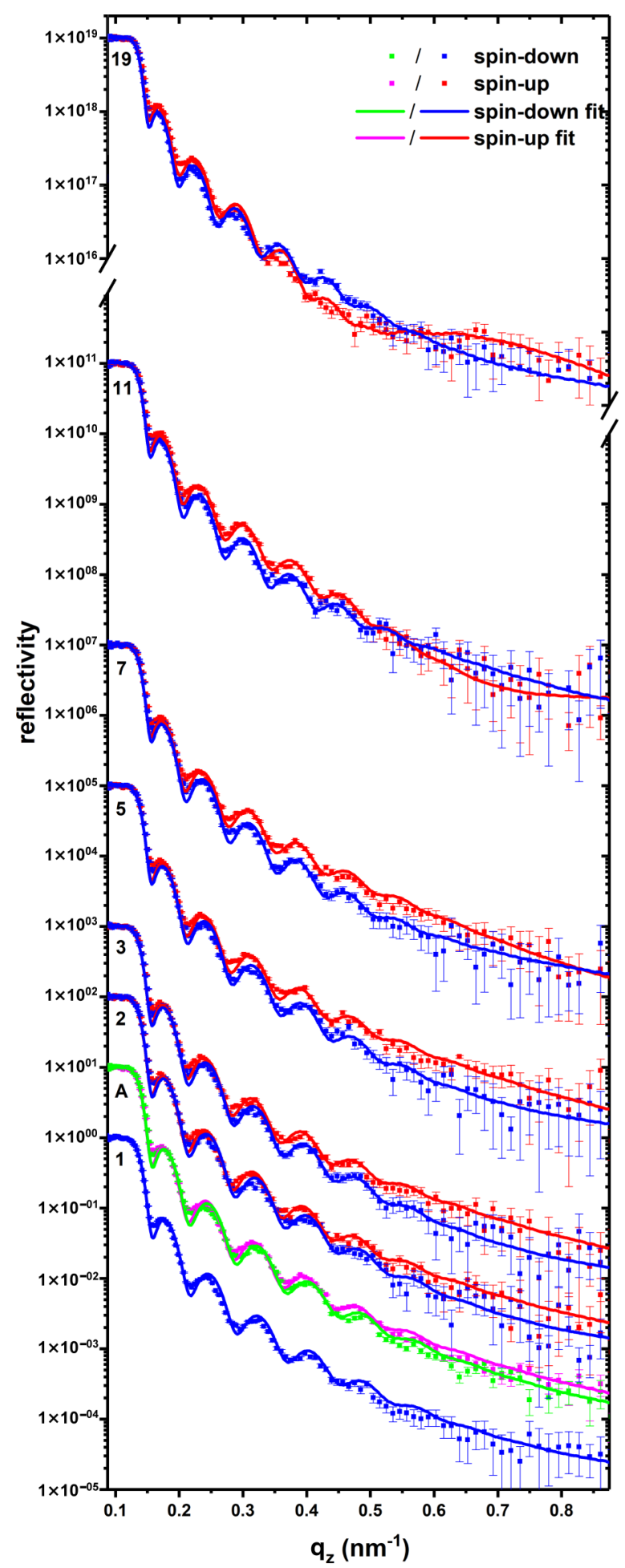

FIG. 2. Measured reflectivity curves are shown in red and blue for spin up and spin down, respectively, together with their best fits, shown as solid lines. The numbers denote the $\mathrm{Pd}$ deposition steps $i$. The letter $A$ denotes the deposition step $i=A$ in which $\mathrm{Fe}$ was deposited. For this deposition step, the spin-up and spin-down reflectivities are shown in magenta and green, respectively. $i=1$ corresponds to the deposition of the Pd underlayer. It was followed by the deposition step with $\mathrm{Fe}(i=A)$. Steps $2 \leqslant i \leqslant 19$ show the reflectivity profiles after deposition of Pd layers on top of the $\mathrm{Fe}$ layer. 


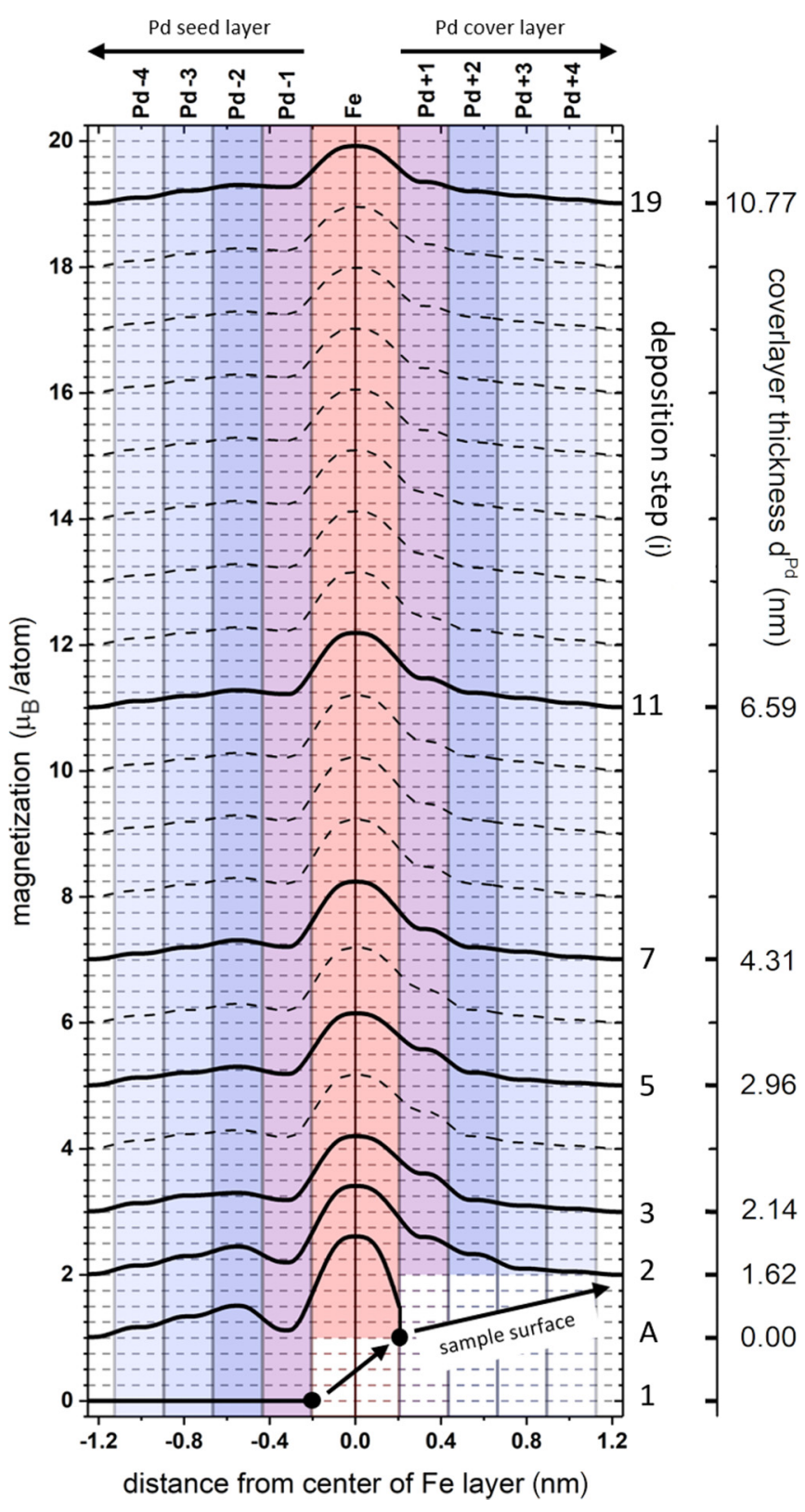

FIG. 3. Magnetization profiles as a function of deposition step and distance from the center of the Fe layer, obtained from the best fit of the profiles of the magnetic scattering length density. Each curve is vertically shifted by $1 \mu_{\mathrm{B}} /$ atom for better visibility. As guide to the eye, the dashed lines show interpolated curves. The magnetization in Pd extends over distances of up to $0.92 \mathrm{~nm}$ to either side of the Fe layer-split up into four magnetic sublayers-and a nonmagnetic rest. The assumed magnetic regions are referred to as $\mathrm{Pd}-4, \mathrm{Pd}-3$, $\mathrm{Pd}-2, \mathrm{Pd}-1(\mathrm{Pd}$ seed layer) and $\mathrm{Pd}+1, \mathrm{Pd}+2, \mathrm{Pd}+3$, and $\mathrm{Pd}+4$ (Pd capping layer). In the initial stages of Pd capping layer growth, a strong side asymmetry for the magnetization of $\mathrm{Pd}-1$ and $\mathrm{Pd}+1$ is observed. This asymmetry vanishes as the structural symmetry of the $\mathrm{Pd} / \mathrm{Fe} / \mathrm{Pd}$ trilayer increases with each deposition step. With increasing symmetry in the magnetization profile, the magnetization in the Fe layer is also slightly reduced.

\section{A. Structural Evolution of the Sample During Growth}

The initial Pd seed layer $(i=1)$ shows a fitted structural thickness of $\sim 72 \mathrm{~nm}$ and an interfacial rms roughness of
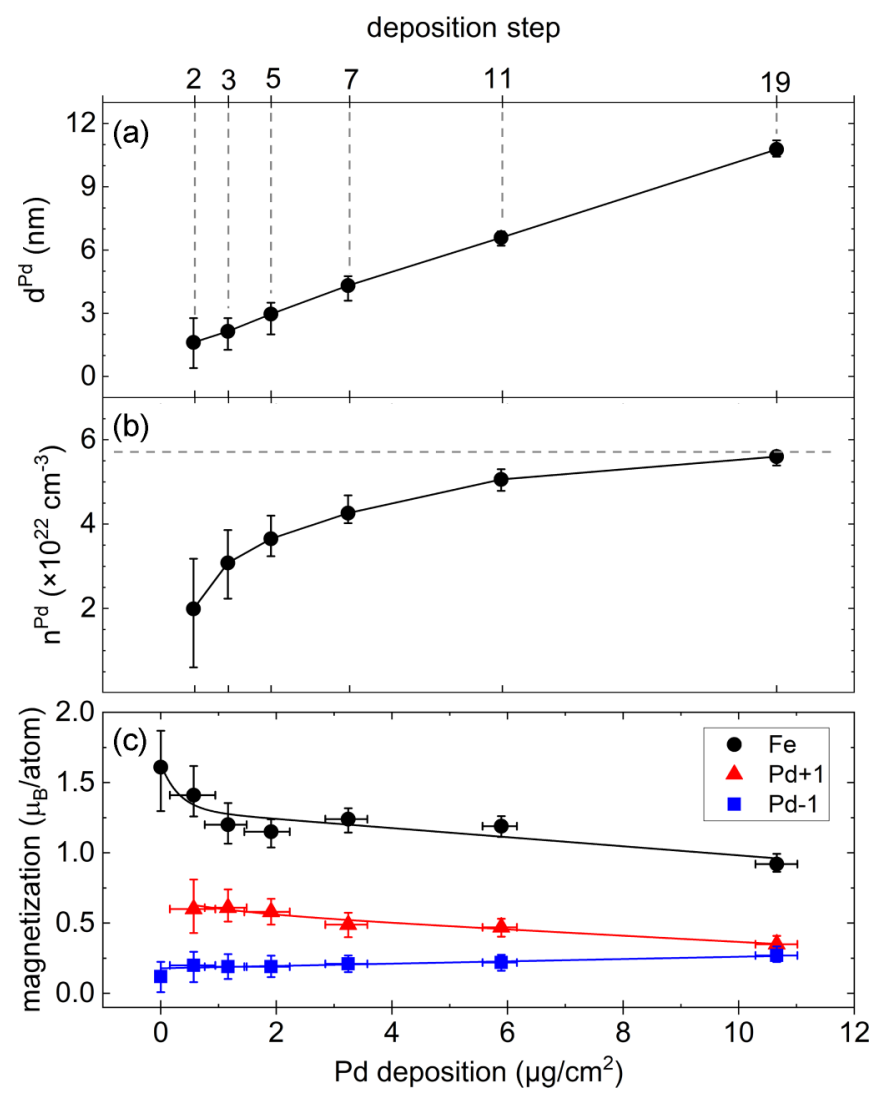

FIG. 4. Evolution of (a) the layer thickness $d^{\mathrm{Pd}}$ and (b) number density $n^{\mathrm{Pd}}$ of the Pd capping layer as a function of deposition step $i$. The connecting lines are a guide to the eye. The dashed line denotes the number density $n^{\text {Pd }_{\text {sed }}} \approx 5.66 \times 10^{22} \mathrm{~cm}^{-3}$ of the underlying Pd seed layer. (c) The magnetization of the $\mathrm{Fe}$ and interfacing Pd layers as a function of the deposited amount of Pd. The line shows a fit to $A_{1} e^{B_{1}\left(x-x_{1}\right)}+A_{2} e^{B_{2}\left(x-x_{2}\right)}+C$.

$\sim 1.8 \mathrm{~nm}$. The Fe layer ( $\operatorname{step} i=A$ ) was found to have a thickness of $\sim 0.4 \mathrm{~nm}$ and a number density of $\sim 8 \times 10^{22} \mathrm{~cm}^{-3}$, which is between the number densities found for liquid $\left(\approx 7.5 \times 10^{22} \mathrm{~cm}^{-3}\right)$ and single-crystalline bulk $(\approx 8.5 \times$ $10^{22} \mathrm{~cm}^{-3}$ ) Fe. The fact that the measured Fe density is $94 \%$ of the bulk single-crystal value is a strong indication that the Fe film is continuous. The $6 \%$ difference may arise from imperfections, including grain boundaries. The continuous structure implied by the PNR results is reproduced in the TEM image, which shows a sharp $\mathrm{Pd} / \mathrm{Fe} / \mathrm{Pd}$ interface and smooth layer contrast. The rms interface roughness of Fe also reproduces the interface roughness of the underlying Pd layer nearly unaltered. The values (Table I) for the Fe film and Pd underlayer agree well with the nominal structure and the expected values for polycrystalline Pd and Fe. Critically, this implies that the $\mathrm{Pd} / \mathrm{Fe}$ interface forms a buried interface: Any Pd capping layer deposition on top of the Fe does not change the structure or the atomic Fe:Pd ratio at the buried interface.

The evolution of the fitting parameters for the Pd capping layer (Fig. 4) indicates a significantly different growth mode compared to the $\mathrm{Fe}$, which formed a continuous layer within the single deposition step. In particular the nuclear scattering length density of the $\mathrm{Pd}$ capping layer was initially much lower than bulk and asymptotically approaches the bulk value 
with increasing thickness. This indicates that the Pd capping layer initially deposits as islands, with a continuously progressing coalescence with each deposition step until a number density value of $\approx 5.7 \times 10^{22} \mathrm{~cm}^{-3}$ is reached. The simultaneous decrease of surface roughness relative to the thickness of the layers in the early growth steps also traces the coalescence of the initial Pd islands. The increasing coalescence, and with it the symmetry of the sample structure relative to the Fe layer, influences the magnetization profile on both sides of the $\mathrm{Fe}$ layer.

\section{B. Magnetism in the Fe layer}

The magnetization profile of the bilayer structure, consisting of the uncapped Fe on Pd (viz., $d^{P d}=0 \mathrm{~nm}$, growth step $i=A$ ), was measured, yielding a converged magnetic moment of $M_{i=A}^{\mathrm{Fe}} \approx 1.6 \mu_{\mathrm{B}} /$ atom $^{\mathrm{Fe}}$. With progressing deposition of the $\mathrm{Pd}$ capping layer onto the $\mathrm{Fe}$ layer, $M^{\mathrm{Fe}}$ successively decreases to a final value of $M_{i=19}^{\mathrm{Fe}} \approx 0.9 \mu_{\mathrm{B}} / \mathrm{atom}^{\mathrm{Fe}}$ (Fig. 3). The lower magnetization of the Fe layer compared to the bulk value of $2.22 \mu_{\mathrm{B}} /$ atom $^{\mathrm{Fe}}$ for bcc Fe may be caused by the Fe layer being too thin to exhibit a bulklike structure and magnetic ordering; an enhanced magnetic moment for $\mathrm{Fe}$ as reported in [15] could not be observed. Note that in [15] PNR was performed with the sample cooled to below $20 \mathrm{~K}$ and that in the model used for the analysis of the PNR data, the Pd was not allowed to carry any induced magnetization. This may explain the discrepancy with the findings presented here. It is noted that a theoretical model with an enhanced magnetic moment within the $\mathrm{Fe}$ alone and without any induced magnetization in Pd cannot reproduce the $i \mathrm{PNR}$ data shown in Fig. 2. The magnetization is well fitted by a pair of exponential functions of the form $A e^{B\left(x-x_{0}\right)}$. This model is consistent with an exponentially dependent interfacial interaction and a density-dependent exchange interaction.

A key observation of $i$ PNR is that the measured saturation magnetization in $\mathrm{Fe}$ decreases with increasing Pd thickness. In the presented measurement configuration, $i \mathrm{PNR}$ is solely sensitive to the net in-plane projection of magnetic moments along the magnetic field direction, and thus, the decrease may be the result of a spin reorientation to the out-of-plane direction or the direction orthogonal to the magnetic field, the formation of domains, or an authentic reduction of the Fe magnetization. Considering the first of these possibilities, an in-plane magnetic field of $70 \mathrm{mT}$ was applied during the measurements to saturate the magnetic moments into the film plane. The decrease in the measured $\mathrm{Fe}$ magnetization could therefore indicate an out-of-plane anisotropy. However, previous works have reported that the $\mathrm{Pd} / \mathrm{Fe}$ system at room temperature has a dominant in-plane anisotropy for all thicknesses of Fe [41], which we expect to remain in plane in the polycrystalline samples here. Other works performed at low temperatures have reported that the out-of-plane anisotropy decreases with increasing thickness of the Pd capping layer [42]. Based on these results, the increasing Pd thickness should increase the in-plane anisotropy, which increases the measured magnetization, contrary to our observations.

This is further supported by considering the structure of the Pd during growth. Specifically, growing the Pd capping layer by room-temperature sputtering, rather than, e.g., e-beam evaporation [42], results in initial island growth with increasing coalescence as more Pd is deposited. The increasing contact area between the $\mathrm{Pd}$ and $\mathrm{Fe}$ at the top interface may change the magnetization. However, as discussed above, the increased interaction should promote an in-plane orientation in the Fe and thus would also increase the measured magnetization, contrary to the observed decrease. We therefore conclude that the observed trend is likely not caused by a spin reorientation away from the field direction due to interfacial perpendicular anisotropy.

Considering now the possibility of domain formation, a reduction in magnetization would imply the spontaneous nucleation of domains against the magnetic field as the Pd capping layer is deposited. This nucleation would incur a penalty in the Zeeman and exchange energies, without any benefit in the magnetostatic or other energy, making it highly unfavorable and unlikely. Furthermore, the 70-mT magnetic field is expected to saturate the Fe film in the in-plane direction.

Finally, we consider an authentic reduction in the Fe magnetization, which may occur due to, e.g., Fe-Pd hybridization. Specifically, as the Pd layer becomes complete, transforming from island growth to a continuous film, an increased hybridization between the Fe-Pd valence electrons occurs. This hybridization will initially scale with the thickness due to the increased number of interfacial neighbors and would result in a modification of the density of states at the Fermi level according to the Stoner model of magnetization. This hybridization alone may be sufficient to explain the decrease in magnetization of the Fe layer. However, as discussed below and as shown in Fig. 3, there are additional changes to the $\mathrm{Pd}$ magnetization; in particular the magnetization at the buried $\mathrm{Pd}$ interface increases with the capping layer thickness, which is inconsistent with hybridization.

We suggest that the decrease in the Fe magnetization is caused by an IDMI which promotes a curling of the magnetization away from a saturated configuration. This deflection would appear as a reduction in the magnetization when probed with PNR.

\section{Magnetism in the Pd layers}

In contrast to most previous studies, $i$ PNR allows simultaneous spatially resolved access to the magnetic properties in both the top and bottom Pd layers, in addition to the Fe layer. The increased spatial resolution is demonstrated to be a great asset, as a strong asymmetry in the induced magnetization is observed in the top versus bottom Pd layers (Fig. 3). The asymmetry is particularly large for very thin Pd capping layers, e.g., step 2, with the proximity-induced magnetism being large and positive on the top surface and much smaller on the bottom. With increasing thickness of the top Pd capping layer, approaching step 19, the magnetization profiles become increasingly symmetric (Fig. 3).

Asymmetry in the magnetism between the top and bottom layers is expected for the initial steps due to the island growth mechanism; however, even after normalizing to the density (Fig. 3), the difference persists. Also the bottom surface is expected to possess the larger and unchanging magnetization for all thicknesses due to its higher number density and buried 
structure. Here, however, the bottom Pd layer possesses the smaller magnetization for all thicknesses of the capping layer, inconsistent with a hybridization model. Interestingly, the proximity-induced magnetism extends $0.92 \mathrm{~nm}$ into the $\mathrm{Pd}$ capping layer, and it is expected that a thickness larger than this will not influence the interfacial magnetism. However, at a Pd thickness of $0.92 \mathrm{~nm}$ the magnetic asymmetry between the top and bottom Pd layers persists and continues to decrease with increasing capping layer thickness. A symmetric magnetization on both sides of the Fe is achieved only once the $\mathrm{Pd}$ capping layer thickness exceeds $\approx 10 \mathrm{~nm}(i \simeq 19)$. This thickness also coincides with achieving a nearly identical nuclear density in each of the Pd layers, demonstrating the strong influence of structure on the magnetization profile.

The strong top/bottom asymmetry in the magnetization of the Pd that we observed for the early growth stages of the Pd capping layer differs from the symmetric magnetization profile presented in [10] where epitaxially grown samples were investigated. However, in [10] the influence of the Fe layer thickness on the magnetization profile and ordering temperatures were the focus of the study, and the initial stages of Pd capping layer growth on the magnetization profile were not investigated. The top/bottom magnetic asymmetry, which is primarily located at the interface, and its dependence on the Pd thickness indicate the presence of proximity-induced magnetism and strongly suggest Dzyaloshinskii-Moriya interactions [29,43]. Specifically, an IDMI can be generated at $\mathrm{HM} / \mathrm{FM}$ interfaces due to spin-orbit coupling and can be strong enough to induce the reorientation of the interfacial moments [44]. The IDMI takes the form $E \propto\left(\vec{S}_{1} \times \vec{S}_{2}\right)$, which motivates neighboring moments to orient at $90^{\circ}$ with a well-defined handedness due to the vector nature of the cross product. As a result of the handedness, at the bottom $\mathrm{Pd} / \mathrm{Fe}$ interface the IDMI will induce a curling of the magnetic moment in a direction determined by the polarity of the DMI coefficient and the orientation of the moments, which is nominally determined by the magnetic field. Although the DMI at the interface of the $3 d / 4 d$ transition metals is weak compared to the $3 d / 5 d$ interfaces, it is not vanishingly small, reported as $-0.1 \mathrm{meV}$ for $\mathrm{Fe} / \mathrm{Pd}$ (compared to $1.7 \mathrm{meV}$ for $\mathrm{Fe} / \mathrm{Ir})$ [43]; the thin $(0.4 \mathrm{~nm}) \mathrm{Fe}$ layer also means that the direct exchange term is significantly suppressed, allowing the IDMI to generate a meaningful effect. Initially, at step 1, the top surface of the $\mathrm{Fe}$ is exposed to vacuum, resulting in a very weak DMI, while the bottom $\mathrm{Pd} / \mathrm{Fe}$ surface does have an IDMI. The asymmetry between the DMI at the top and bottom surfaces results in a curling of the magnetization, particularly at the interface. We note that the magnetization in the $\mathrm{Pd}$ is present only due to proximity effects and therefore strongly emulates the interfacial magnetization. Once more, the presented PNR results measure only the net projection of the magnetization along the direction of the guide field. The curling of the interfacial moments away from the field direction therefore manifests as a reduced magnetization. Subsequent deposition of a $\mathrm{Pd}$ capping layer forms a top $\mathrm{Fe} / \mathrm{Pd}$ interface, which in turn generates an equal and opposite IDMI due to the handedness of the interaction. As the IDMIs from the top and bottom interfaces cancel out, the exchange interaction dominates, which symmetrizes the proximity-induced magnetization. Indeed, as the Pd capping layer thickness is increased, the magnetization at the $\mathrm{Pd} / \mathrm{Fe}$ interface increases to agree with the expected value.

Performing a simple analytical check reveals that the DMI should be reasonably present in the system. Specifically, the DMI is in direct competition with the exchange interaction and the Zeeman energy. Considering the scale of these energies per site, the DMI is $0.1 \mathrm{meV}$, while the Zeeman is $\approx 0.004 \mathrm{meV}$ in the 70 -mT applied field. The exchange interaction for bulk Fe is $\approx 850 \mathrm{meV}$ but will be weaker in this unitcell-thick film due to the fewer neighbors. The competition between the DMI and exchange will therefore dominate, and the resultant magnetization will possess a small, progressive canting of the moments, which appears as a reduction in the macroscopic magnetization. The combined effects of a weaker DMI and thin Fe results in a DMI to exchange ratio approximately $30 \%$ that of a thicker Ir/Fe system.

In detail, for our polycrystalline sample, after the Fe deposition step, the magnetization $M_{\mathrm{i}=\mathrm{A}}^{\mathrm{Pd}-1}$ at the interface from Fe to the underlying Pd layer is comparatively small, showing a value of $M_{i=A}^{\mathrm{Pd}-1}=0.12 \mu_{\mathrm{B}} /$ atom $^{\mathrm{Pd}-1}$. Upon deposition of the $\mathrm{Pd}$ capping layer the induced magnetism in the $\mathrm{Pd}$ underlayer increases to $M_{i=19}^{\mathrm{Pd}-1} \approx 0.3 \mu_{\mathrm{B}}$ atom $^{\mathrm{Pd}-1}$ at its final thickness. In comparison, the induced magnetism in the $\mathrm{Pd}$ capping layer $M^{\mathrm{Pd}+1}$ evolves opposite to $M^{\mathrm{Pd}-1}$ : With the first $\mathrm{Pd}$ deposition step, a magnetization of $M_{i=2}^{\mathrm{Pd}+1} \approx 0.6 \mu_{\mathrm{B}} /$ atom $^{\mathrm{Pd}+1}$ is observed, which gradually decreases with layer thickness to a final value of $M_{i=19}^{\mathrm{Pd}+1} \approx 0.3 \mu_{\mathrm{B}}$ atom $^{\mathrm{Pd}+1}$.

Comparing these values with the literature, for the fully grown sample $(i=19)$ the interfacial moment agrees well with previous works, which report an induced moment of $0.32 \mu_{\mathrm{B}}-0.38 \mu_{\mathrm{B}} /$ atom $^{\mathrm{Pd}}$ for ideal interfaces $[13,15,20]$. Interestingly, Ref. [15] also reports a moment of $0.54 \mu_{\mathrm{B}} / \mathrm{atom}^{\mathrm{Pd}}$ for imperfect interfaces. This value agrees well with the initial deposition (e.g., step 2), in which we observe an induced moment of $\approx 0.6 \mu_{\mathrm{B}} /$ atom $^{\mathrm{Pd}+1}$. This result is consistent with our expectation that the $\mathrm{Pd}$ film initially exhibits an island growth mode, effectively corresponding to a high surface roughness. Furthermore, moments of $0.17 \mu_{\mathrm{B}} /$ atom $^{\mathrm{Pd}}$ [20] and $0.26 \mu_{\mathrm{B}} /$ atom $^{\mathrm{Pd}}$ [13] were reported for the second monolayer. These findings also agree well with the result of our in situ study for the fully grown sample if only the Pd capping layer is included in the investigations.

\section{SUMMARY AND CONCLUSIONS}

Using in situ polarized neutron reflectometry, the evolution of the magnetism in a polycrystalline $\operatorname{Pd}(11 \mathrm{~nm}) /$ $\mathrm{Fe}(0.41 \mathrm{~nm}) / \operatorname{Pd}(72 \mathrm{~nm})$ trilayer heterostructure was investigated during growth. An induced magnetization was observed in $\mathrm{Pd}$ up to $0.92 \mathrm{~nm}$ from the Fe interface. Without any $\mathrm{Pd}$ capping layer (viz., only $\mathrm{Pd} / \mathrm{Fe}$ ), there is a small magnetic moment of $\approx 0.12 \mu_{B} /$ atom $^{\mathrm{Pd}}$ induced in the $\mathrm{Pd}$ underlayer $\left(\mu_{\mathrm{B}} /\right.$ atom $\left.^{\mathrm{Pd}-1}\right)$. Concurrently, the Fe magnetization is $1.6 \mu_{\mathrm{B}} /$ atom $^{\mathrm{Fe}}$, surprisingly small compared with the bcc Fe bulk value of $2.2 \mu_{\mathrm{B}} /$ atom $^{\mathrm{Fe}}$ [45]. Subsequent deposition of a $\mathrm{Pd}$ capping layer further reduces the measured $\mathrm{Fe}$ moment to a final value of $\approx 0.9 \mu_{\mathrm{B}} /$ atom $^{\mathrm{Fe}}$. This reduction is accompanied by an increase of the induced moment in the $\mathrm{Pd}$ underlayer at the interface, a decrease of the induced moment in the Pd 
capping layer, and an increase in the symmetry of the $\mathrm{Pd} / \mathrm{Fe} / \mathrm{Pd}$ magnetization profile. Interestingly, the symmetry of the magnetization profile is influenced by the Pd capping layer well beyond the interface, in regions which do not show induced magnetization themselves. Complementary TEM imaging of the Fe/Pd shows sharp interfaces, which indicates that the magnetization does not result from interdiffusion of $\mathrm{Fe}$ and $\mathrm{Pd}$ at the interface.

Our observations as a whole indicate the presence of two effects in the investigated polycrystalline $\operatorname{Pd}(11 \mathrm{~nm}) /$ $\mathrm{Fe}(0.41 \mathrm{~nm}) / \mathrm{Pd}(72 \mathrm{~nm})$ trilayer heterostructure, namely, (i) proximity-induced magnetism extending into the $\mathrm{Pd}$ up to approximately $1 \mathrm{~nm}$ on both sides of the $\mathrm{Pd} / \mathrm{Fe}$ interface and (ii) a Dzyaloshinskii-Moriya interaction located directly at the $\mathrm{Pd} / \mathrm{Fe}$ interface. The observations are similar as in the $\mathrm{Co} / \mathrm{Pt}$ system, described in [29], where no correlation between the presence of proximity-induced magnetism and Dzyaloshinskii-Moriya interaction was reported. However, as was shown here, the dominance of either effect and the magnetization for the Pd underlayer and Fe layers, respectively, can experimentally be influenced by restoring the structural and electronic symmetry.

\section{ACKNOWLEDGMENTS}

This work is based on experiments performed at the Swiss spallation neutron source (SINQ), Paul Scherrer Institute, Villigen, Switzerland. The funding of this project by the Deutsche Forschungsgemeinschaft (DFG) within the Transregional Collaborative Research Center TRR 80 "From electronic correlations to functionality" is gratefully acknowledged. We thank B. Hjörvarsson (Uppsala University) for fruitful discussions.
[1] S. Ikeda, K. Miura, H. Yamamoto, K. Mizunuma, H. D. Gan, M. Endo, S. Kanai, J. Hayakawa, F. Matsukura, and H. Ohno, A perpendicular-anisotropy $\mathrm{CoFeB}-\mathrm{MgO}$ magnetic tunnel junction, Nat. Mater. Lett. 9, 721 (2010).

[2] R. O. Cherifi, V. Ivanovskaya, L. C. Phillips, A. Zobelli, I. C. Infante, E. Jacquet, V. Garcia, S. Fusil, P. R. Briddon, N. Guiblin, A. Mougin, A. A. Ünal, F. Kronast, S. Valencia, B. Dkhil, A. Barthelemy, and M. Bibes, Electric-field control of magnetic order above room temperature, Nat. Mater. Lett. 13, 354 (2014).

[3] X. R. Wang, C. J. Li, W. M. Lü, T. R. Paudel, D. P. Leusink, M. Hoek, N. Poccia, A. Vailionis, T. Venkatesan, J. M. D. Coey, E. Y. Tsymbal, Ariando, and H. Hilgenkamp, Imaging and control of ferromagnetism in $\mathrm{LaMnO}_{3} / \mathrm{SrTiO}_{3}$ heterostructures, Science 349, 716 (2015).

[4] N. Reyren, S. Thiel, A. D. Caviglia, K. L. Fitting, G. Hammerl, C. Richter, C. W. Schneider, T. Kopp, A.-S. Rüetschi, D. Jaccard, M. Gabay, D. A. Muller, J.-M. Triscone, and J. Mannhart, Superconducting interfaces between insulating oxides, Science 317, 1196 (2007).

[5] A. Brinkman, M. Huijben, M. van Zalk, J. Huijben, U. Zeitler, J. C. Maan, W. G. van der Wiel, G. Rijnders, D. H. A. Blank, and H. Hilgenkamp, Magnetic effects at the interface between non-magnetic oxides, Nat. Mat. Lett. 6, 493 (2007).

[6] J. F. Gregg, Spintronics: A growing science, Nat. Mater. 6, 798 (2007).

[7] A. Schmehl, V. Vaithyanathan, A. Herrnberger, S. Thiel, C. Richter, M. Liberati, T. Heeg, M. Röckerath, L.-F. Kourkoutis, S. Mühlbauer, P. Böni, D. A. Muller, Y. Barash, J. Schubert, Y. Idzerda, J. Mannhart, and D. G. Schlom, Epitaxial integration of the highly spin-polarized ferromagnetic semiconductor $\mathrm{EuO}$ with silicon and GaN, Nat. Mater. 6, 882 (2007).

[8] X. Z. Yu, N. Kanazawa, Y. Onose, K. Kimoto, W. Z. Zhang, S. Ishiwata, Y. Matsui, and Y. Tokura, Near room-temperature formation of a skyrmion crystal in thin-films of the helimagnet FeGe, Nat. Mater. 10, 106 (2011).

[9] S. X. Huang and C. L. Chien, Extended Skyrmion Phase in Epitaxial FeGe(111) Thin Films, Phys. Rev. Lett. 108, 267201 (2012).

[10] T. P. A. Hase, M. S. Brewer, U. B. Arnalds, M. Ahlberg, V. Kapaklis, M. Björck, L. Bouchenoire, P. Thompson, D. Haskel,
Y. Choi, J. Lang, C. Sánchez-Hanke, and B. Hjörvarsson, Proximity effects on dimensionality and magnetic ordering in Pd/Fe/Pd trialyers, Phys. Rev. B 90, 104403 (2014).

[11] Z. Celinski, B. Heinrich, J. F. Cochran, W. B. Muir, A. S. Arrott, and J. Kirschner, Growth and Magnetic Studies of Lattice Expanded Pd in Ultrathin $\mathrm{Fe}(001) / \mathrm{Pd}(001) / \mathrm{Fe}(001)$ Structures, Phys. Rev. Lett. 65, 1156 (1990).

[12] C. Liu and S. D. Bader, Morphology of Fe/Pd(100) films studied using photoemission from physisorbed Xe, Phys. Rev. B 44, 2205 (1991).

[13] J. Vogel, A. Fontaine, V. Cros, F. Petroff, J.-P. Kappler, G. Krill, A. Rogalev, and J. Goulon, Palladium magnetism in $\mathrm{Pd} / \mathrm{Fe}$ multilayers studied by XMCD at the $\mathrm{PdL}_{2,3}$ edges, J. Magn. Magn. Mater. 165, 96 (1997).

[14] J. Childress, R. Kergoat, O. Durand, J.-M. George, P. Galtier, J. Miltat, and A. Schuhl, Magnetic properties and domain structure of epitaxial (001) Fe/Pd superlattices, J. Magn. Magn. Mater. 130, 13 (1994).

[15] E. E. Fullerton, D. Stoeffler, K. Ounadjela, B. Heinrich, Z. Celinski, and J. A. C. Bland, Structure and magnetism of epitaxially strained $\mathrm{Pd}(001)$ films on $\mathrm{Fe}(001)$ : Experiment and theory, Phys. Rev. B 51, 6364 (1995).

[16] O. Rader, E. Vescovo, J. Redinger, S. Blügel, C. Carbone, W. Eberhardt, and W. Gudat, Fe-Induced Magnetization of Pd: The Role of Modified Pd Surface States, Phys. Rev. Lett. 72, 2247 (1994).

[17] S.-K. Lee, J.-S. Kim, B. Kim, Y. Cha, W. K. Han, H. G. Min, J. Seo, and S. C. Hong, Surface alloying and magnetism of ultrathin Fe films on Pd(001), Phys. Rev. B 65, 014423 (2001).

[18] M. Pärnaste, M. Marcellini, E. Holmström, N. Bock, J. Fransson, O. Eriksson, and B. Hjörvarsson, Dimensionality crossover in the induced magnetization of Pd layers, J. Phys.: Condens. Matter 19, 246213 (2007).

[19] Z.-P. Shi and B. M. Klein, Interlayer magnetic coupling of multilayer structures with palladium spacer layers, Phys. Rev. B 52, 12516 (1995).

[20] S. Blügel, B. Drittler, R. Zeller, and P. H. Dederichs, Magnetic properties of $3 \mathrm{~d}$ transition metal monolayers on metal substrates, Appl. Phys. A 49, 547 (1989).

[21] A. Soumyanarayanan, M. Raju, A. L. Gonzalez Oyarce, A. K. C. Tan, M.-Y. Im, A. Petrović, P. Ho, K. H. Khoo, 
M. Tran, C. K. Gan, F. Ernult, and C. Panagopoulos, Tunable room-temperature magnetic skyrmions in $\mathrm{Ir} / \mathrm{Fe} / \mathrm{Co} / \mathrm{Pt}$ multilayers, Nat. Mater. 16, 898 (2017).

[22] S. Tacchi, R. E. Troncoso, M. Ahlberg, G. Gubbiotti, M. Madami, J. Åkerman, and P. Landeros, Interfacial Dzyaloshinskii-Moriya Interaction in Pt/CoFeB Films: Effect of the Heavy-Metal Thickness, Phys. Rev. Lett. 118, 147201 (2017).

[23] X. Ma, G. Yu, C. Tang, X. Li, C. He, J. Shi, K. L. Wang, and X. Li, Interfacial Dzyaloshinskii-Moriya Interaction: Effect of $5 d$ Band Filling and Correlation with Spin Mixing Conductance, Phys. Rev. Lett. 120, 157204 (2018).

[24] R. Tolley, S. A. Montoya, and E. E. Fullerton, Roomtemperature observation and current control of skyrmions in $\mathrm{Pt} / \mathrm{Co} / \mathrm{Os} / \mathrm{Pt}$ thin films, Phys. Rev. Mater. 2, 044404 (2018)

[25] R. Streubel, C.-H. Lambert, N. Kent, P. Ercius, A. T. N'Diaye, C. Ophus, S. Salahuddin, and P. Fischer, Experimental evidence of Chiral ferrimagnetism in amorphous GdCo films, Adv. Mater. 30, 1800199 (2018).

[26] P. Kuświk, M. Matczak, M. Kowacz, K. Szuba-Jabłoński, N. Michalak, B. Szymański, A. Ehresmann, and F. Stobiecki, Asymmetric domain wall propagation caused by interfacial Dzyaloshinskii-Moriya interaction in exchange biased $\mathrm{Au} / \mathrm{Co} / \mathrm{NiO}$ layered system, Phys. Rev. B 97, 024404 (2018).

[27] E. C. Corredor, S. Kuhrau, F. Kloodt-Twesten, R. Frömter, and H. P. Oepen, SEMPA investigation of the DzyaloshinskiiMoriya interaction in the single, ideally grown $\mathrm{Co} / \mathrm{Pt}(111)$ interface, Phys. Rev. B 96, 060410(R) (2017).

[28] R. M. Rowan-Robinson, A. A. Stashkevich, Y. Roussigné, M. Belmeguenai, S.-M. Chérif, A. Thiaville, T. P. A. Hase, A. T. Hindmarch, and D. Atkinson, The interfacial nature of proximity-induced magnetism and the DzyaloshinskiiMoriya interaction at the Pt/Co interface, Sci. Rep. 7, 16835 (2017).

[29] H. Yang, A. Thiaville, S. Rohart, A. Fert, and M. Chshiev, Anatomy of Dzyaloshinskii-Moriya Interaction at Co/Pt Interfaces, Phys. Rev. Lett. 115, 267210 (2015).

[30] A. Soumyanarayanan, N. Reyren, A. Fert, and C. Panagopoulos, Emergent phenomena induced by spin-orbit coupling at surfaces and interfaces, Nature (London) 539, 509 (2016).

[31] J. Stahn and A. Glavic, Focusing neutron reflectometry: Implementation and experience on the TOF-reflectometer Amor, Nucl. Instrum. Methods Phys. Res., Sect. A 821, 44 (2016).

[32] F. Ott, Neutron surface scattering. Application to magnetic thin films, Collect. SFN 13, 02004 (2014).
[33] H. Zabel and K. Theis-Bröhl, Polarized neutron reflectivity and scattering studies of magnetic heterostructures, J. Phys.: Condens. Matter 15, S505 (2003).

[34] A. Schmehl, T. Mairoser, A. Herrnberger, C. Stephanos, S. Meir, B. Förg, B. Wiedemann, P. Böni, J. Mannhart, and W. Kreuzpaintner, Design and realization of a sputter deposition system for the in situ- and in operando-use in polarized neutron reflectometry experiments, Nucl. Instrum. Methods Phys. Res., Sect. A 883, 170 (2018).

[35] W. Kreuzpaintner, B. Wiedemann, J. Stahn, J.-F. Moulin, S. Mayr, T. Mairoser, A. Schmehl, A. Herrnberger, P. Korelis, M. Haese, J. Ye, M. Pomm, P. Böni, and J. Mannhart, In-situ Polarized Neutron Reflectometry: Epitaxial Thin Film Growth of Fe on $\mathrm{Cu}(001)$ by dc Magnetron Sputtering, Phys. Rev. Appl. 7, 054004 (2017).

[36] J. Stahn, T. Panzner, U. Filges, C. Marcelot, and P. Böni, Study on a focusing, low-background neutron delivery system, Nucl. Instrum. Methods Phys. Res., Sect. A 634, S12 (2011).

[37] J. Stahn, U. Filges, and T. Panzner, Focusing specular neutron reflectometry for small samples, Eur. Phys. J. Appl. Phys. 58, 11001 (2012)

[38] J. Stahn and A. Glavic, Efficient polarization analysis for focusing neutron instruments, J. Phys.: Conf. Ser. 862, 012007 (2017).

[39] F. Ott, SimulReflec, version 1.72, http://www-llb.cea.fr/prism/ programs/simulreflec/simulreflec.html.

[40] M. Björck and G. Andersson, GenX: An extensible X-ray reflectivity refinement program utilizing differential evolution, J. Appl. Crystallogr. 40, 1174 (2007).

[41] C. Liu and S. D. Bader, Two-dimensional magnetic phase transition of ultrathin iron films on Pd(100), J. Appl. Phys. 67, 5758 (1990).

[42] T. Ueno, M. Sawada, K. Furumoto, T. Tagashira, S. Tohoda, A. Kimura, S. Haraguchi, M. Tsujikawa, T. Oda, H. Namatame, and $\mathrm{M}$. Taniguchi, Interface atomic structures and magnetic anisotropy of $\mathrm{Fe}$ and $\mathrm{Pd} / \mathrm{Fe}$ monatomic films on Pd(001), Phys. Rev. B 85, 224406 (2012).

[43] B. Dupé, M. Hoffmann, C. Paillard, and S. Heinze, Tailoring magnetic skyrmions in ultra-thin transition metal films, Nat. Commun. 5, 4030 (2014).

[44] S. Seki, Y. Okamura, K. Kondou, K. Shibata, M. Kubota, R. Takagi, F. Kagawa, M. Kawasaki, G. Tatara, Y. Otani, and Y. Tokura, Magnetochiral nonreciprocity of volume spin wave propagation in chiral-lattice ferromagnets, Phys. Rev. B 93, 235131 (2016).

[45] J. Kübler, Magnetic moments of ferromagnetic and antiferromagnetic bcc and fcc iron, Phys. Lett. A 81, 81 (1981). 